\title{
RETOURNEMENT TEMPOREL ITÉRATIF : CONVERGENCE DU PROCESSUS
}

\author{
C. PRADA, N. CHAKROUN, J.L. THOMAS, F. WU et M. FINK \\ Laboratoire Ondes et Acoustique, Université Paris 7, ESPCI, 10 rue Vauquelin, F-75005 Paris, \\ France
}

\begin{abstract}
RESUME- Cet article expose une méthode de focalisation sélective à travers des milieux inhomogènes, sur des objets échogènes complexes. Elle utilise un miroir ultrasonore à retournement temporel fonctionnant en mode itératif. On y présente quelques éléments de théorie ainsi que des exemples numériques et expérimentaux mettant en évidence la convergence du processus.

ABSTRACT- A selective focusing method for complexe reflective targets is presented. It uses an iterative time reversal mirror working in the pulse echo mode. A matrix theory is developped and some numerical and experimental results are given in order to demonstrate the convergence of the process.
\end{abstract}

Introduction: L'efficacité du retournement temporel pour focaliser sur une cible échogène a déjà été mise en évidence ${ }^{[1]-[4]}$. La méthode consiste à transformer par retournement temporel une onde issue de la cible en une onde convergeant vers la cible. Elle utilise un réseau de transducteurs ultrasonores pouvant fonctionner en émission ou en réception. Chaque transducteur est commandé par un émetteur programmable couplé à une mémoire électronique. Le processus de retournement temporel comprend trois phases: (1) - Le réseau de transducteurs émet une première onde incidente pour exciter la cible. (2) - L'onde réfléchie par celle-ci est captée par le réseau et les signaux correspondants sont numérisés. (3) - Les signaux sont appliqués aux transducteurs après une inversion de leur chronologie. Ceci génère une deuxième onde incidente qui est la retournée temporelle de l'onde réfléchie.

Lorsque le milieu contient un réflecteur unique, la deuxième onde incidente converge vers celui-ci. Mais lorsque la cible est constituée de réflecteurs répartis sur une zone de dimensions supérieures à celles de la cellule de résolution du réseau, cette onde n'est pas toujours focalisée de façon optimale. Afin de concentrer le maximum d'énergie sur l'un des réflecteurs, on peut itérer le processus. En effet, le choix de la première onde incidente est libre. En particulier, la deuxième onde incidente décrite précédemment peut être utilisée comme première onde incidente d'un nouveau processus de retournement temporel. L'opération peut être répétée plusieurs fois, la deuxième onde incidente d'un processus devenant la première onde incidente du processus suivant, c'est le retournement temporel itératif. Cette méthode permet une focalisation sélective vers le réflecteur dominant.

En général, les caratéristiques des réflecteurs ne sont pas les seuls paramètres déterminant l'issue de l'itération. Celles du réseau de transducteurs jouent aussi un rôle important et prédire l'issue du processus itératif n'est pas toujours immédiat. Afin de déterminer la convergence de ce processus, nous avons mis au point un modèle matriciel dans l'approximation de la diffusion simple. Le modèle: Il permet le calcul du champ de pression acoustique en chacun des réflecteurs à une itération donnée, à partir du champ en tous les réflecteurs à l'itération précédente. Il tient compte de la géométrie du réseau de transducteurs, de la réponse acousto-électrique des transducteurs et 
des caractéristiques des réflecteurs.

La réponse acousto-électrique en émission et en réception: On admet que les transducteurs utilisés fonctionnent en mode piston, c'est à dire qu'ils fournissent une vitesse uniforme sur leur face avant. De plus ils sont supposés réversibles et linéaires. Ces hypothèses se traduisent de la façon suivante: (1) - à l'émission, la vitesse de vibration du transducteur se déduit du signal électrique qui lui est appliqué par convolution avec sa réponse acousto-électrique en émission notée $e(t),(2)$ - à la réception, le signal électrique délivré par le transducteur est la convolution de la force acoustique reçue avec la réponse acousto-électrique en réception notée $r(t)$.

La réponse impulsionnelle de diffraction: Elle traduit les effets de l'ouverture du transducteur et est notée $h(\overrightarrow{\mathbf{r}}, t)$; en émission, elle représente la valeur du potentiel acoustique pour une excitation de vitesse normale à la surface du transducteur de forme temporelle $\delta(t)$. La propagation étant réversible, $h(\overrightarrow{\mathbf{r}}, t)$ est aussi la force acoustique perçue par le transducteur poúr une onde de pression impulsionnelle $\delta(t)$ issue du point $\overrightarrow{\mathbf{r}}$.

Les réflecteurs: On suppose que la cible est constituée de $d$ réflecteurs ponctuels situés en $\overrightarrow{\mathbf{r}}_{1}, \overrightarrow{\mathbf{r}}_{2}, \ldots, \overrightarrow{\mathbf{r}}_{d}$ de coefficients $\kappa_{1}, \kappa_{2}, \ldots, \kappa_{d}$. Pour la pression incidente $p(\overrightarrow{\mathbf{r}}, t)$, un réflecteur situé en $\overrightarrow{\mathbf{r}}_{i}$ se comporte comme une source ponctuelle d'amplitude $\kappa_{i} \frac{d^{2}}{d t^{2}} p\left(\overrightarrow{\mathbf{r}}_{i}, t\right)$.

Le réseau est constitué de $L$ transducteurs $\left(E_{1}, \ldots, E_{L}\right)$. On note $h_{l_{i}}(t)$ la réponse impulsionnelle du transducteur $E_{l}$ au point $\overrightarrow{\mathbf{r}}_{i}$. Lorsque chaque $E_{l}$ est excité par le signal $g_{l}(t)$, pour $1<l<L$, le champ au point réflecteur $\overrightarrow{\mathbf{r}}_{i}$ s'écrit $p\left(\overrightarrow{\mathbf{r}}_{i}, t\right)=e(t) \underset{t}{\otimes} \sum_{l=1}^{L} g_{l}(t) \underset{t}{\otimes h_{l i}}(t)$

La boucle d'itération: On notera $p_{1}^{n}(t), p_{2}^{n}(t), \ldots . ., p_{d}^{n}(t)$ les pressions reçues par les d réflecteurs après le $\mathrm{n}$-ième retournement temporel. Les ondes réfléchies par les $d$ réflecteurs sont captées par les L éléments $E_{l}$. La diffusion multiple étant négligée, le signal reçu par le transducteur $E_{l}$ s'exprime comme suit

$$
g_{l}^{n}(t)=\sum_{i=1}^{d} r(t) \underset{t}{\otimes} h_{l i}(t) \underset{t}{\otimes} \kappa_{i} \frac{d^{2}}{d t^{2}} p_{i}^{n}(t)
$$

L'excitation simultanée des L éléments du réseau par les L signaux électriques $g_{l}^{n}(T-t)$ conduit au champ de pression au point $\overrightarrow{\mathbf{r}}_{j}$

$$
p_{j}^{n+1}(t)=e(t) \underset{t}{\otimes r}(T-t) \otimes \sum_{l=1}^{L} \sum_{i=1}^{d} h_{l j}(t) \underset{t}{\otimes} \kappa_{i} h_{l i}(T-t) \underset{t}{\otimes} \frac{d^{2}}{d t^{2}} p_{i}^{n}(T-t)
$$

Le calcul d'une itération dans le domaine temporel nécessite donc $d^{2}$ calculs de convolution. En passant dans l'espace dual de Fourier, on peut écrire l'opérateur d'itération sous forme matricielle. Ecriture matricielle: Par transformée de Fourier temporelle de l'équation (2) on obtient

$$
P_{j}^{n+1}(\omega)=-\omega^{2} e^{3 j \omega T} E(\omega) R^{*}(\omega) \sum_{l=1}^{L} \sum_{i=1}^{d} H_{l j}(\omega) \kappa_{i} H_{l i}^{*}(\omega) P_{i}^{n *}(\omega) \quad \text { pour } \quad 1 \leq j \leq d
$$

Soient $\mathrm{P}^{n}(\omega)$ le vecteur de composantes $P_{1}^{n}(\omega), P_{2}^{n}(\omega), \ldots, P_{d}^{n}(\omega)$ et $A(\omega)$ le complexe $-\omega^{2} e^{3 j \omega T} E(\omega) R^{*}(\omega)$, l'équation (3) se met alors sous forme matricielle, en omettant la dépendance en $\omega$, il vient

$$
\mathrm{P}^{n+1}=A \mathrm{KP}^{n *}
$$




$$
\operatorname{avec} \quad \mathrm{K}=\left(\begin{array}{cccc}
\kappa_{1} \sum_{l=1}^{L}\left|H_{l 1}\right|^{2} & \kappa_{2} \sum_{l=1}^{L} H_{l 1} H_{l 2}^{*} & \ldots & \kappa_{d} \sum_{l=1}^{L} H_{l 1} H_{l d}^{*} \\
\kappa_{1} \sum_{l=1}^{L} H_{l 2} H_{l 1}^{*} & \kappa_{2} \sum_{l=1}^{L}\left|H_{l 2}\right|^{2} & \ldots & \kappa_{d} \sum_{l=1}^{L} H_{l 2} H_{l d}^{*} \\
\vdots & \vdots & \ddots & \vdots \\
\kappa_{1} \sum_{l=1}^{L} H_{l d} H_{l 1}^{*} & \kappa_{2} \sum_{l=1}^{L} H_{l d} H_{l 2}^{*} & \ldots & \kappa_{d} \sum_{l=1}^{L}\left|H_{l d}\right|^{2}
\end{array}\right)
$$

On peut écrire $\mathrm{K}={ }^{t} H H^{*} \mathrm{D}$ avec $\mathrm{H}=\left(H_{i l}\right)_{1 \leq i \leq d, 1 \leq l \leq L}$ et $\mathrm{D}=\left(\kappa_{i} \delta_{i j}\right)_{1 \leq i \leq d, 1 \leq j \leq d}$.

$\mathrm{H}$ est la matrice $(L \times d)$ de l'opérateur de propagation et $\mathrm{D}$ la matrice diagonale $(d \times d)$ de l'opérateur de diffusion. Le vecteur de pression $\mathrm{P}^{n}$ s'exprime alors en fonction de $\mathrm{P}^{o}$ de la façon suivante

$$
\mathrm{P}^{2 n}=\left(K^{*}\right)^{n} \mathrm{P}^{o} \quad \text { et } \quad \mathrm{P}^{2 n+1}=\left(K K^{*}\right)^{n} \mathrm{KP}^{o *}
$$

Une fois la matrice $K$ évaluée, le calcul d'une itération à une fréquence donnée se ramène à une conjugaison et à un produit matriciel. En effectuant ce calcul à toutes les fréquences, on obtient par transformée de Fourier inverse les signaux de pression reçus par les réflecteurs. Le cycle d'itération est représenté sur la figure 1.

Interprétation physique de la matrice $K$ : Le coefficient $k_{i j}$ de $K$ est le signal reçu par le réflecteur $j$ lorsque l'on focalise sur le réflecteur $i$ par retournement temporel en l'absence des autres réflecteurs. Il traduit le couplage entre les réflecteurs $i$ et $j$ vis à vis du retournement temporel. Ce couplage est d'autant plus faible que les deux réflecteurs sont bien résolus par le réseau de transducteurs, il s'observe en comparant les termes non diagonaux aux termes diagonaux: si les réflecteurs sont suffisamment espacés, ce sont les coefficients diagonaux qui dominent et l'itération du processus conduit à une focalisation vers le réflecteur de plus fort coefficient diagonal. Dans le cas contraire, les couplages sont importants et il est plus difficile de prévoir l'issue d'une itération.

On présente trois exemples obtenus avec des cibles constituées de deux objets résolus par le réseau de transducteurs.

Exemple numérique: Une barrette de 64 transducteurs sert de miroir à retournement temporel. Deux objets spéculaires sont modélisés par deux groupes de réflecteurs ponctuels (barres verticales sur la figure 2). On a calculé le champ de pression dans le plan des cibles après 1, 2, 4 et 6 retournements temporels (figure 2). La première onde incidente choisie favorise le petit objet de telle sorte qu'à la première itération celui-ci reçoit la pression maximale. Cependant, dès la deuxième itération c'est le front d'onde convergeant vers l'objet le plus large qui domine. A la sixième itération, l'onde focalise parfaitement sur celui-ci.

\section{Résultats expérimentaux}

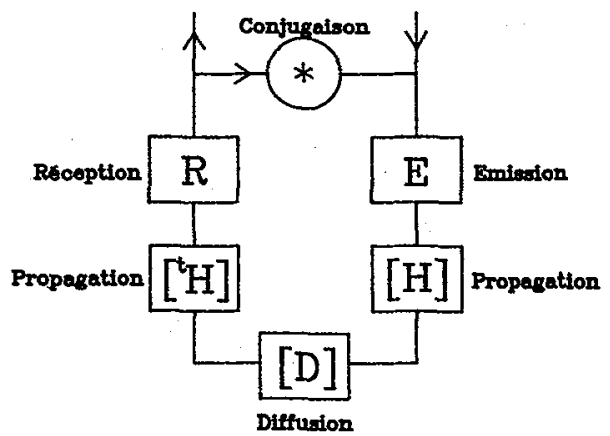

Figure 1

1 - Dans la première expérience, le réseau est une barrette plane de 64 éléments. La cible est constituée de deux fils perpendiculaires à la barrette, observés à travers un aberrateur en caoutchouc. On a représenté le champ de pression après $1,2,4$ et 6 retournements temporels (figure 3 ). L'onde est d'abord focalisée sur les deux diffuseurs en dépit de l'aberrateur et elle finit par converger vers le réflecteur le plus fort. 
2 - La deuxième expérience est réalisée avec une matrice de 25 transducteurs. La cible est composée de deux lithiases biliaires. Le champ de pression est mesuré pour quatre itérations (figure 3). A la première, on observe deux maxima à l'emplacement des deux calculs. A la quatrième, le faisceau converge vers le plus réflecteur.

Champ de pression dans le plan de la cible au cours des itérations successives.
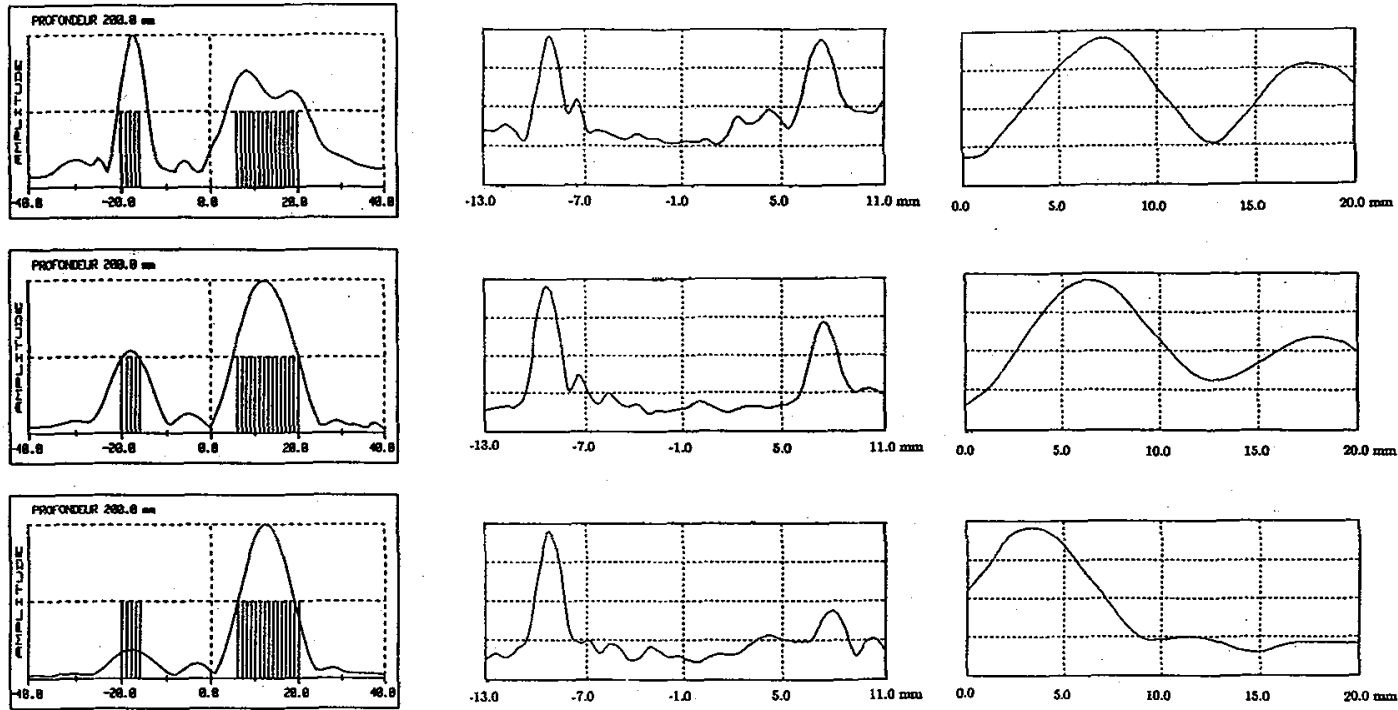

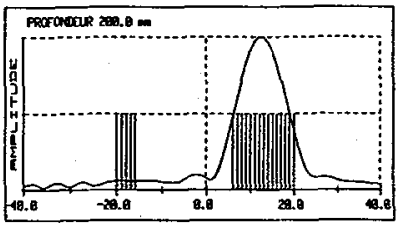

Figure 2

Simulation

Itérations $1,2,4$ et 6

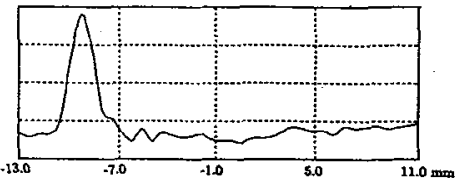

Figure 3

Expérience 1

Itérations 1,2,4 et 6

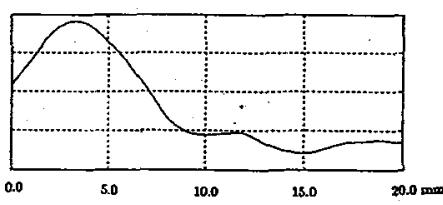

Figure 4

Expérience 2

Itérations $1,2,3$ et 4

Conclusion: Nous avons démontré lạ convergence du processus de retournement temporel itératif sur une cible composée de 2 réflecteurs séparés par le réseau de transducteurs. La théorie prévoit que l'itération conduit à focaliser sélectivement vers le réflecteur dominant de la cible. Elle est validée par deux expériences, l'une réalisée à travers une milieu aberrateur et l'autre sur une cible complexe composée de deux lithiases biliaires. L'application de cette méthode au contrôle non-destructif et à la lithotripsie est à l'étude.

\section{Références}

[1] "Self-focusing in homogeneous media with time reversal acoustic mirrors", M.Fink, C.Prada, F.Wu and D.Cassereau, Proc. IEEE Ultrasonics Symposium 681-686, (1989).

[2] "Autofocalisation par miroir à renversement temporel", C. Prada, F.Wu et M.Fink, Colloque de Physique, C2-361, 1er CFA Fév 90.

[3] "The iterative time reversal mirror: A solution to self-focusing in the pulse echo mode", C.Prada, F.Wu and M.Fink, J. Acoust. Soc .Am. 90 (2) 1119-1129 (1991).

[4] "Le retournement temporel des ondes ultrasonores: application à la focalisation", C.Prada, Thèse de Doctorat, Juin 1991. 